\title{
Influence of embedded through section connectors on the behavior of a new strengthening technique for concrete structures \\ VALENTE Tiago ${ }^{1, a}$, COLARUSSO Davide ${ }^{2, b}$, PECCE Marisa ${ }^{3, c}$, CERONI Francesca $^{4, \mathrm{~d}^{*}}$, BARROS Joaquim ${ }^{5, \mathrm{e}}$
}

\author{
${ }^{1}$ PhD Student, CiviTest V.N. Famalicão, Portugal, \\ ${ }^{2}$ Civil Engineer, University of Sannio, Benevento, Italy \\ ${ }^{3}$ Full Professor, University of Sannio, Benevento, Italy \\ ${ }^{4}$ Associate Professor, University of Napoli Parthenope, Napoli, Italy \\ ${ }^{3}$ Full Professor, ISISE, Univ. Minho, Guimarães, Portugal \\ atiagovalente@civitest.com, bdavid5f06@live.it, cpecce@unisannio.it, \\ dfrancesca.ceroni@uniparthenope.it, ebarros@civil.uminho.pt
}

Keywords: concrete structures, infill walls, connectors, ultra-high ductile fiber reinforced mortar

\begin{abstract}
An innovative technique is being developed for the structural rehabilitation of Reinforced Concrete (RC) structures. In particular, the infill walls of RC framed structures are often identified as non-structural elements, but currently are considered with an important role in the structural behavior because they participate to the in-plane strength and stiffness of the frames and they can give very dangerous crashes out-of-plane. In this paper a strengthening technique aimed to repair infill walls is proposed. It is based on the application of outer thin layers of ultra-high ductile fiber reinforced mortar (UHDFRM) applied according to the shotcrete technique, including the use of embedded through section (ETS) connectors. This strengthening system can exhibit a high strength and ductile behavior, increase the load carrying capacity, energy absorption and dissipation capacities, and ultimately improve the structural response of RC structures when submitted to loading conditions typical of seismic events. An experimental program was outlined in order to assess the contribution of different types of ETS connectors on the behavior of the strengthening system. The experimental program comprised the performance of push-out tests on samples representative of the structural strengthening solution, namely low strength concrete samples. The experimental results are discussed in detail in order to highlight the effectiveness of the various types of ETS connectors tested.
\end{abstract}

\section{Introduction}

Buildings with reinforced concrete (RC) frames in-filled with masonry walls are a very common type of construction, with a worldwide dissemination. In the design process, the structural contribution of masonry walls in RC frames is usually not taken into account.

Although the common disregard for the effect of infill walls in the structural analysis of buildings, the recently published Eurocode 8 states that the frame-infill interaction should be take into account during the structural analysis. Additionally, appropriate measures should be taken in consideration to avoid brittle failure and premature disintegration of the infill walls, as well as the partial or total out-of-plane collapse of slender masonry panels [1].

Evidence has also been found that the contribution of unreinforced infill walls for the behavior of RC frames is greatly compromised when submitted to reversed cyclic loading, such is the case of earthquake actions. This is due to the low strength, brittleness, low energy dissipation capacity and rapid stiffness degradation of the unreinforced masonry walls [2]-[5]. However, if both frame and 
infill are designed to be ductile, then the stiffness degradation and strength deterioration under cyclic loading can be minimized.

In the scope of a research project "InoTec - Innovative material of ultra-high ductility for the rehabilitation of the built environment", that involves a private research company - CiviTest - and the University of Minho, is being developed a new material for structural rehabilitation of buildings, namely for strengthening of infill walls of RC frame type structures.

The new strengthening material, designated in this paper as "Ultra-high ductile fiber reinforced mortar" (UHDFRM), is a cement based material with relatively high percentage of fibers to achieve a ultra-high ductility in tension. The UHDFRM has the particularity of presenting tensile failure for strain levels relatively high $(>1 \%)$, with the formation of diffuse multiple cracks patterns, and having a high energy absorption capacity.

The structural rehabilitation technique is based in the application of thin outer layers of UHDFRM, applied by projection, on infill walls. The strengthening layers are connected, by the use of embedded through section (ETS) connectors made of fiber reinforced polymers (FRP), that are immune to corrosion effects. In this way, the strengthening system performs like a sandwich panel, with outer layers of UHDFRM that exhibit a high strength and ductile behavior, and high durability. This technique increases the in- and out-of-plane load carrying capacity of the infill walls, as well as the energy absorption and dissipation capacities, and ultimately improve the structural response of RC structures when submitted to loading conditions typical of seismic events.

The already performed mechanical characterization of the UHDFRM [6] revealed that in flexural tests the material presents an high tensile strength and a high energy absorption capacity when compared to a commercially available strengthening mortar. The study also pointed that the UHDFRM has elasticity and strength levels that are compatible with infill walls made of ceramic bricks and other low strength substrates, assuring a good bond between the strengthening and strengthened materials.

The present work intends to assess the contribution of different types of ETS connectors on the behavior of the strengthening system, by the performance of push-out tests on samples representative of the structural strengthening solution.

\section{Experimental program}

\section{Prototypes}

Although the presented technique was mainly developed for strengthening masonry walls, in the present experimental program were built prototypes with low strength concrete substrates. This decision was taken, in order to avoid the possibility of existence of multiple failure modes that could exist with prototypes of masonry walleyes substrate, which would increase the test complexity and could undermine the interpretation of the push-out tests results and undermine the main objective of this works - assess the influence of the different types of ETS connectors in the structural system.

The prototypes substrate were made of very low strength concrete cubes ( $f_{c}=7.2 \mathrm{MPa}$ at 28 days), at which were applied outer layers of UHDFRM with approximately $2.5 \mathrm{~cm}$ of thickness, in two opposite faces of the cubes. In the center of the prototype was applied a ETS connector, with the extremities hooked in the UHDFRM layers, that intercepts all the prototype layers. The scheme and geometry of the prototypes is presented in Figure 1a.

In order to achieve the geometric regularity of the prototypes, in the application procedure of the UHDFRM layers were used molds. Besides the wetting of the concrete cube faces, no surface treatment was applied to the concrete cubes.

The configuration and constitution of the prototypes assures the structural simplicity of the push-out test and limits the existence of different failure modes (failure of the interface between the UHDFRM), while keeping the strengthening solution representativeness. 
The ETS connectors used are made of different materials: steel bars, with Ø8mm (type A500NR) and high adherence surface; fiber glass (GFRP) bars, with Ø8mm; and a hybrid connector formed with Eglass fiber filaments and polyester resin (Figure 1b). The hybrid type of connector was specifically developed for this application. In the present study, were used two models of the hybrid connector with 20 and 50 filaments of E-glass fibers (F20/F50). In the group of the tested ETS connectors, the hybrid types are the ones with lower shear stiffness $\left(E_{F 50}>E_{F 20}\right)$, followed by the GFRP connectors $(E=50 G P a)$ and the steel connectors $(E=200 G P a)$. The hybrid connectors were designed to develop a high adherence to the UHDFRM layers, due to the inclusion of hooked ends formed by Ø8mm GFRP bars (Figure 1b). The remaining connectors present straight ends, although is expectable that the steel connectors have a better adherence to UHDFRM than the GFRP bars, due to the high adherence surface of the steel bars used.

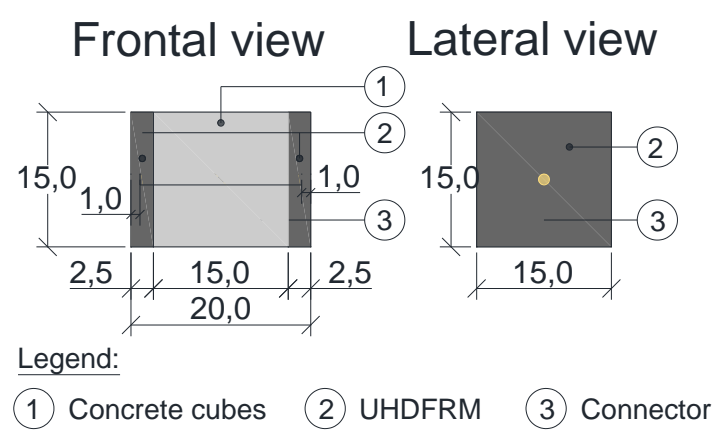

a)



b)

Figure 1. a) Lay-out and geometry of specimens; b) Example of hybrid connectors.

Briefly, the manufacture process of the prototypes is: step 1: fabrication and cure of the concrete cube; step 2: drilling a hole in the center of the concrete cube, for introduction of the ETS connector (Figure 2a); step 3: filling of the voids between the connector and the concrete cube with high strength grout; step 4: application and cure of the outer layers of UHDFRM (Figure 2b).



a)



b)

Figure 2. a) Position of ETS connector in the specimen; b) Specimen with UHDFRM layers.

\section{Push-out tests setup}

The push-out test is based on the testing procedure presented in the Annex B of Eurocode 4 [7]. This test consists in the application of an axial load in the core of the prototype (concrete cube), which from the shear strength of the connectors and the adherence between the UHDFRM layers and the concrete cube transmits the load to the outer layers of UHDFRM, whose displacement are restrained at the base. The test setup is presented in Figure 3a). The load application system is formed by a hydraulic group, a servo-actuator and a load cell with a maximum capacity of $250 \mathrm{kN}$. The test is performed in displacement control, with a velocity of the actuator of $0.02 \mathrm{~mm} / \mathrm{s}$. The test ends when the first of the following events occurs: total loss of the load carrying capacity of the prototypes; vertical displacement 
of the concrete cube reaches $20 \mathrm{~mm}$. During the test, the slip and opening of the prototypes interface is monitored with 4 LVDT's. The LVDT's positioning is presented in Figure 3b). The tests were performed in the laboratory of CiviTest.

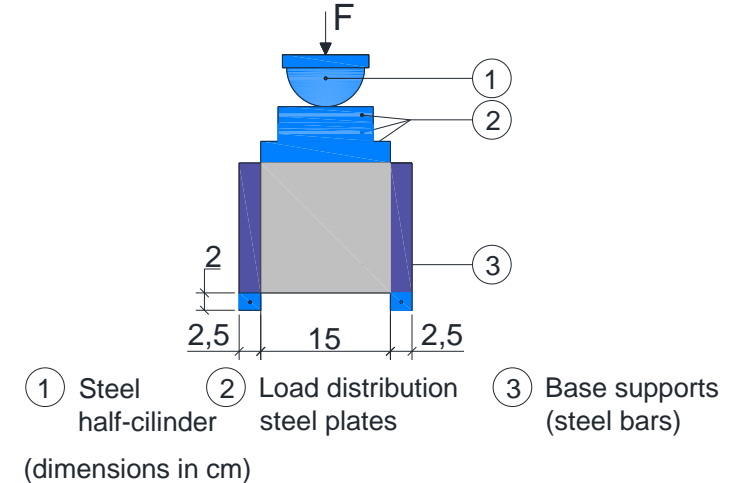

a)

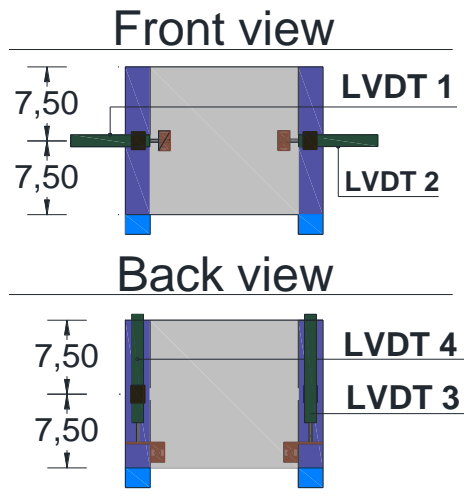

b)

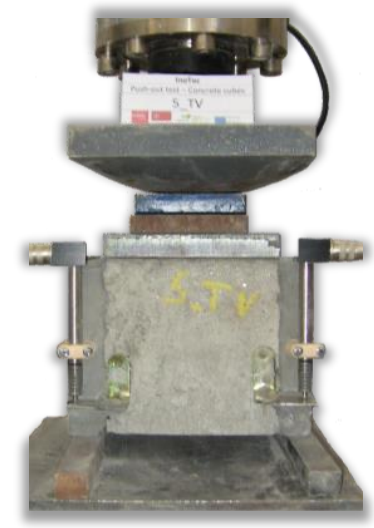

c)

Figure 3. a) Setup of the push-out test; b) LVDT's positioning; c) Prototype ready to be tested.

\section{Discussion of experimental results}

In the following section are presented the results of the push-out tests performed at 13 prototypes, from which 1 without any connector; 2 with steel connectors; 3 with GFRP connectors; and 7 with hybrid connectors (4 with F20 model and 3 with F50 model). In Figure 4a is presented the typical crisis due to the failure of the interfaces between the UHDFRM layers and the concrete cube. In some prototypes, the failure developed in only one interface, while at the other interface no significant deformation was recorded. This type of failure had higher incidence in the prototypes with GFRP and F20 hybrid connectors, due to their lower shear stiffness compared with the steel ones. Moreover, it was detected that the failure zone included a thin layer of concrete substrate (Figure 4b), due to its low strength. This fact highlights that, even without any surface treatment, the UHDFRM exhibits a very good adherence to low strength substrates, as is the case of masonry and low strength concrete walls. In all tested prototypes failure in the UHDFRM layers was never detected, while a total rupture of the hybrid connectors near the interface zone of the prototypes occurred (Figure 4c). On the other hand, the steel and GFRP connectors kept its integrity until the end of the tests.

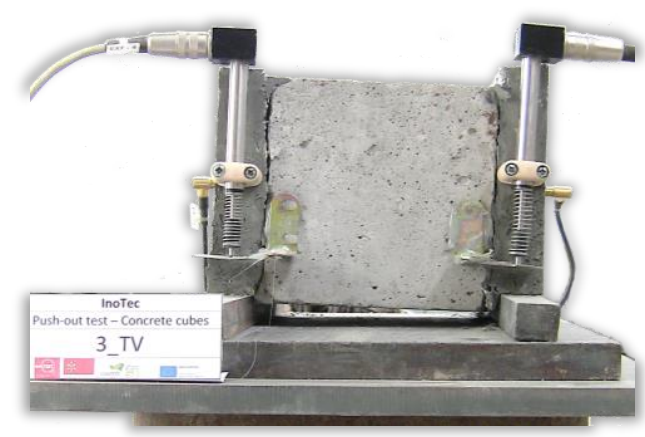

a)



b)



c)

Figure 4. a-b) Typical interface failure of the prototypes; c) Failure of the hybrid connector.

In Figure 5 and 6 are presented, respectively, the load-slip and load-opening relationships of the failure interface between the UHDFRM layer and concrete substrate. It is possible to denote that the prototype without connector exhibits a very fragile behavior. In opposition, the prototypes with connectors exhibit a post-peak ductile response, namely the samples with steel, GFRP and F50 hybrid connectors. 
The prototypes with GFRP connectors presented the least pronounced decay of load carrying capacity of the interface in the post-peak range of the tests. It is possible to identify that for a load level of around $20 \mathrm{kN}$ it was detected a reduction of stiffness in the load-slip and load-opening response of the interface. This load level must coincide with the crack initiation stage of the interface, resulting in the deterioration of the bond properties between the UHDFRM and the concrete substrate, and to the stage where the connectors starts to be solicited.

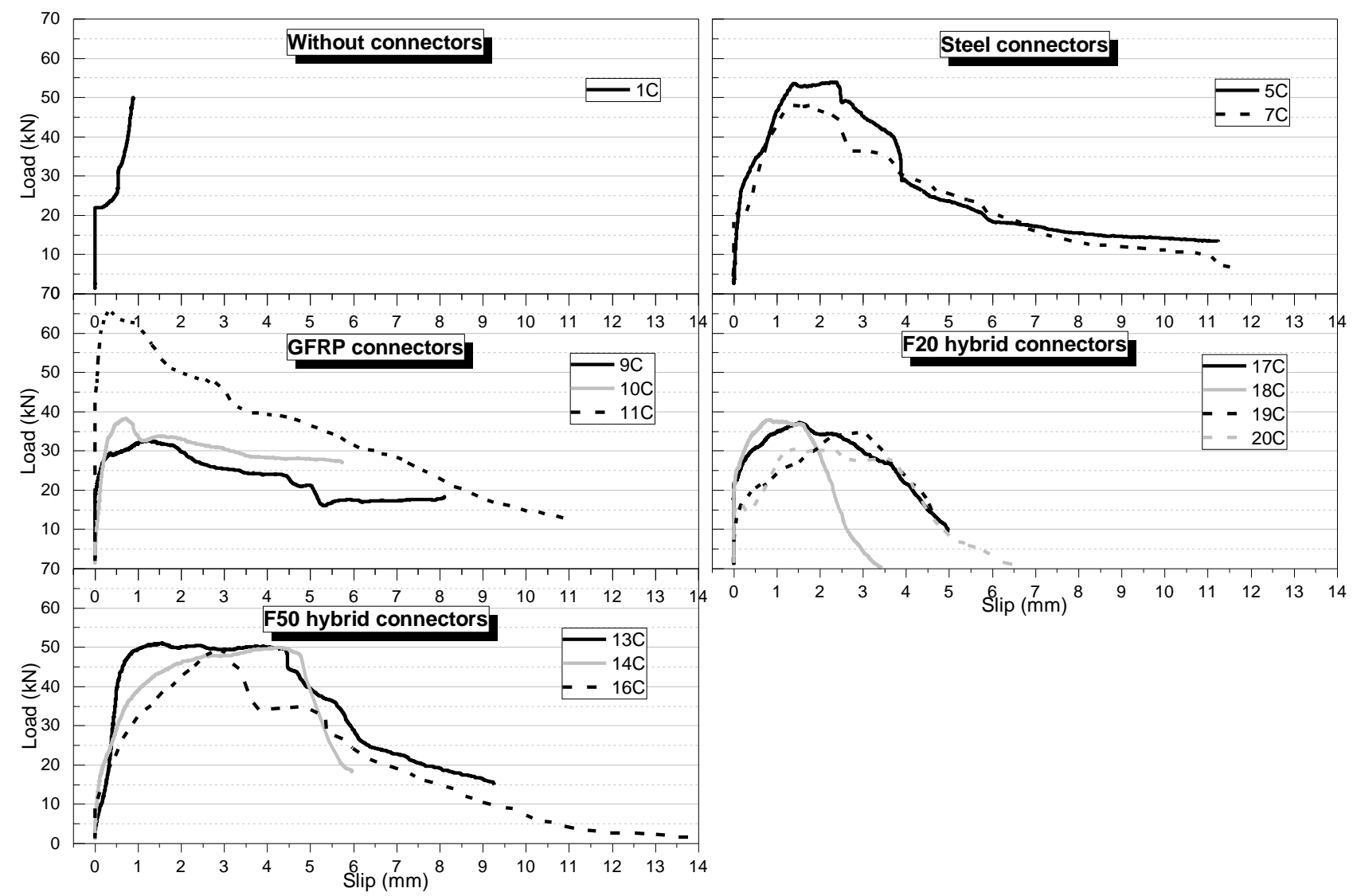

Figure 5. Load-slip relationship at concrete/UHDFRM interface for different connectors.

In Figure 7 is presented the comparison of the average values of the maximum load registered during the push-out tests of the prototypes with different types of connectors. The prototypes with steel, GFRP and F50 hybrid connectors, as well as the prototypes without connectors, presented very similar average maximum load values. The prototypes with F20 hybrid connectors presented slightly lower mean maximum load values. In fact, it is possible to state that the use of ETS connectors did not improve the load carrying capacity of the prototypes.

In Figure 8 is presented a comparison of the average values of the slip of the interface corresponding to the maximum load (a) and the ultimate slip values registered in the push-out tests (b). The use of connectors produces a significant enhancement of the deformation capacity of the prototypes in the pre- and post-peak stage of the load-slip response of the interface. In this context, the steel and the F50 hybrid connectors stand out for enabling the higher increase of slip deformation in the pre- and postpeak stage. In the pre-peak stage, the increase of slip deformation is particularly noticeable for the prototypes with steel and hybrid connectors. In other hand, the prototypes with GFRP connectors just revealed an increase of slip deformation in the post-peak stage, as in the pre-peak stage the slip deformation is similar to the prototypes without connectors. The increase of the interface slip capacity of the prototypes with hybrid connectors can be justified by the low shear stiffness of these elements. This behavior is particularly noticeable in the loading phase, between the crack initiation $(20 \mathrm{kN})$ and the maximum applied load, where it is registered a significant increase of slip deformation (Figure 5). 
In respect to the steel connectors, due to its higher stiffness, in the deformation process a localized damaged can be induced in the concrete matrix and UHDFRM in the surrounding zones of the connectors, enhancing the slip deformation of the interface, mainly for high levels of the connector deformation.

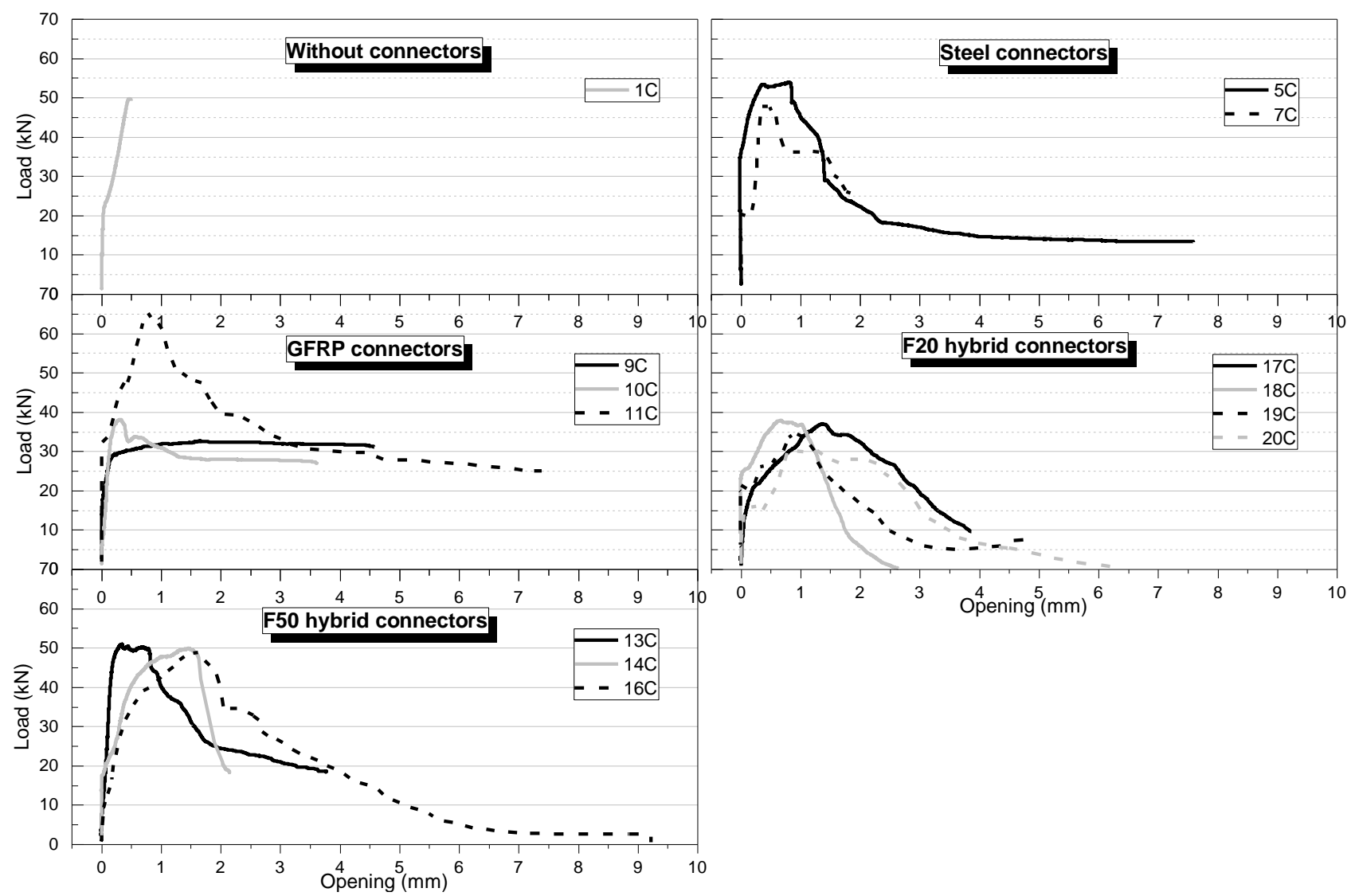

Figure 6. Load-opening relationship at concrete/UHDFRM interface for different connectors.

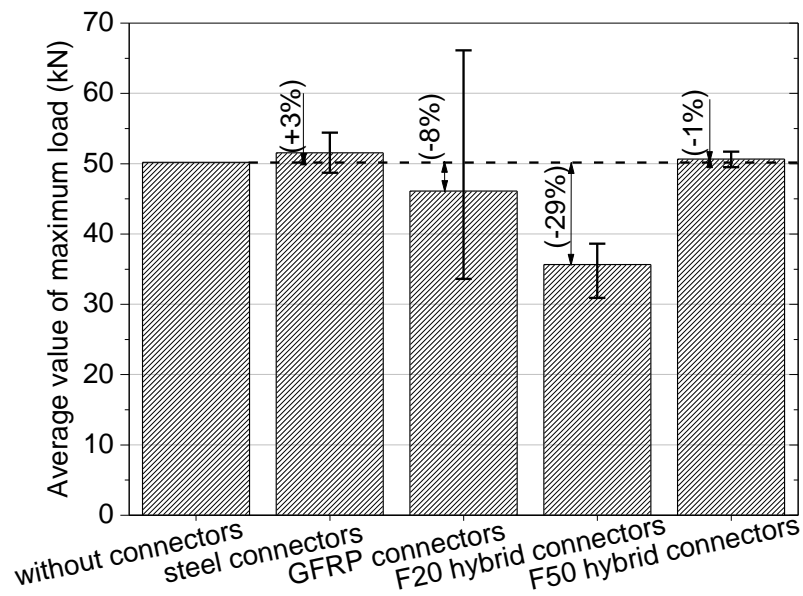

Figure 7. Average value of the maximum load for the prototypes with different types of connectors.

In Figure 9 the relationship between the sliding and opening of the interface at maximum load is compared. It is possible to state that the prototypes with hybrid and steel connectors present a higher slip deformation than opening deformation of the interface. These connectors present, indeed, a high adherence to the UHDFRM, enhancing the restriction for the interface to open during the test. Moreover, as previously discussed, the high interface slip is justified for the hybrid connectors by the low shear stiffness of these elements, while for the steel connectors by localized damages induced in 
the surrounding concrete and UHDFRM layer. In other hand, the GFRP connectors present very similar slip and opening deformation levels, exhibiting a balanced relationship between the shear stiffness and the adherence with the UHDFRM for the tested prototypes.

In Figure 10 the average values of the absorption energy from the interface slip (a), calculated until a maximum slip deformation of $5 \mathrm{~mm}$ is reached, and from the interface opening (b), calculated until a maximum opening deformation of $3 \mathrm{~mm}$ is reached, are plotted. For all the prototypes, the absorption energy from the interface slip deformation is higher than the absorption energy from its opening. The dissipated energy with the interface slip is very similar for the prototypes with steel, GFRP and F50 hybrid connectors. In other hand, the prototypes with GFRP connectors exhibited the highest absorption energy in the opening deformation of the interface, which could be justified by the lower adherence between the GFRP connector and the UHDFRM layer, allowing a higher deformability and subsequent dissipation of energy from the interface opening.

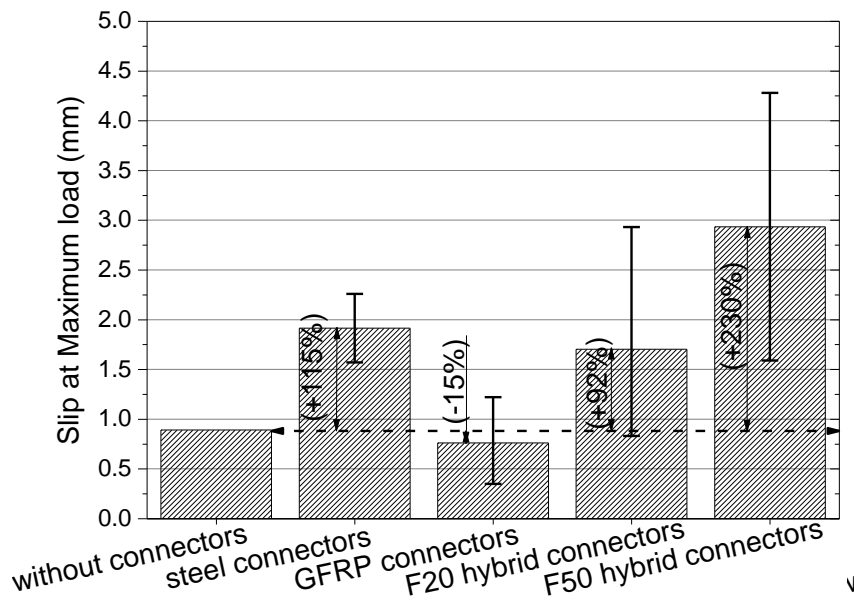

a)

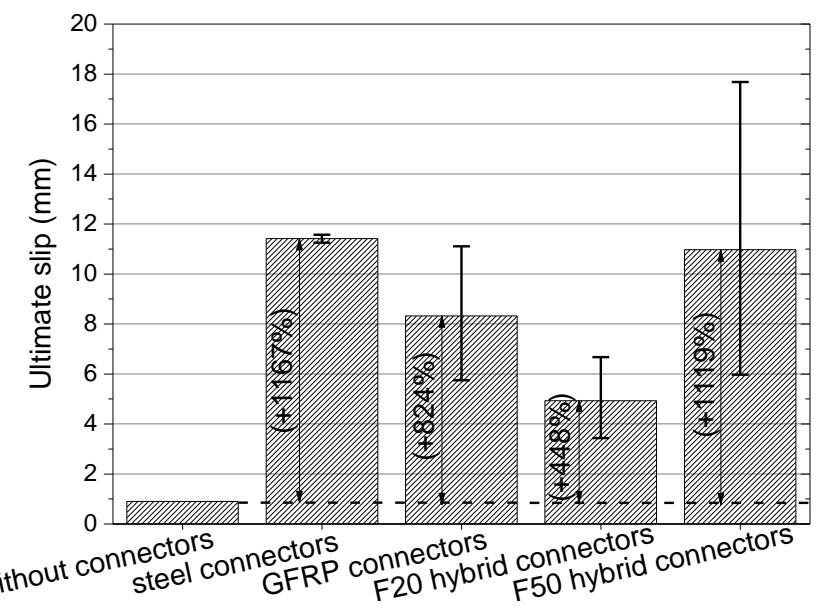

b)

Figure 8. Average values of slip at maximum load (a) and at ultimate slip (b).

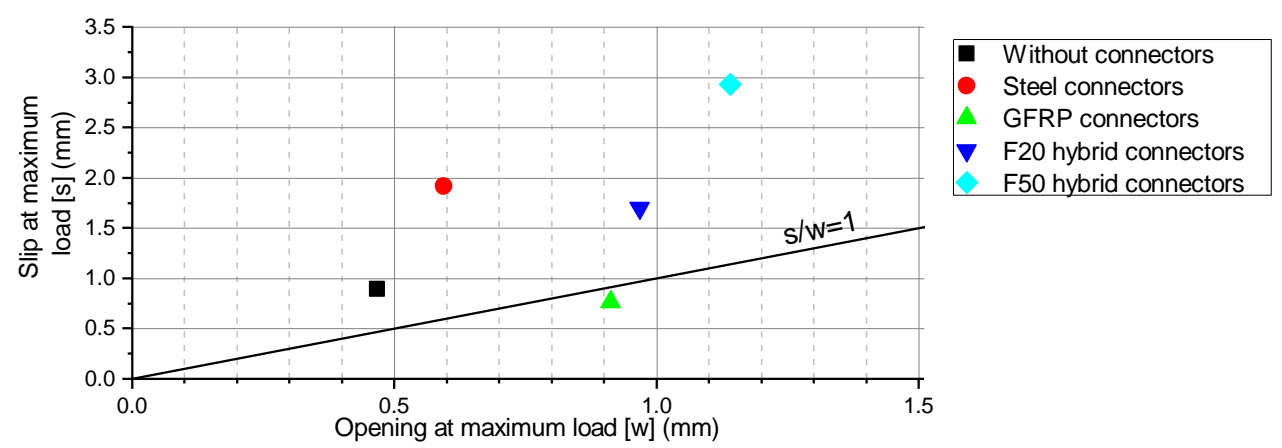

Figure 9. Slip vs. opening of the interface at maximum load for the different connectors.

\section{Conclusions}

In the present work the results of push-out tests on low strength concrete cubes strengthened by outer thin layers of ultra-high ductile fiber reinforced mortar (UHDFRM) including embedded through section (ETS) connectors are discussed. Such a new technique is pointed to repair infill walls and the experimental tests herein discussed are aimed to assess the effectiveness of different types of ETS connectors (glass and steel bars, hybrid connectors made of glass fiber filaments and polyester resin) on the shear strength of the UHDFRM layers/concrete interface.

The experimental tests highlighted that the failure zone includes a thin layer of concrete substrate, which indicates that, even without any surface treatment, the UHDFRM layer presents an adequate adherence and compatibility to low strength concrete substrates. It was also evidenced that the ETS 
connectors did not promote an increase of the load carrying capacity, but a significant increase of the deformation capacity of the interface. The steel and hybrid connectors promoted a higher slip than opening deformation of the interface, as result of the high adherence of both types of connectors to the UHDFRM layer, of the low shear stiffness of the hybrid connectors, and of the localized damages induced by the steel connectors in the surrounding concrete and matrix. The GFRP connectors presented similar levels of slip and opening interface deformation, revealing an adequate balance between the adherence and shear stiffness. However, in terms of absorption, all types of prototypes exhibited a higher energy dissipation from the interface slip than from its opening.

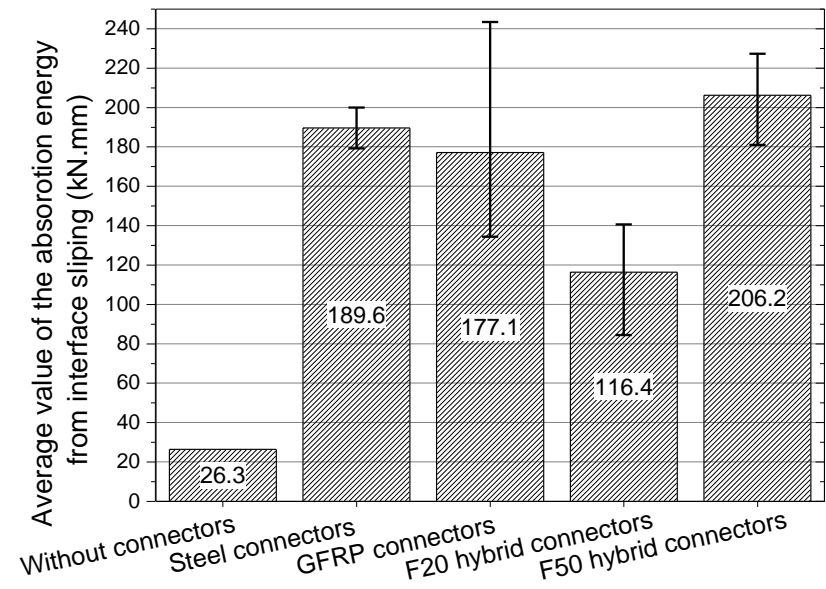

a)



b)

Figure 10. Average absorption energy from the interface slip (a) and opening (b) for different connectors.

\section{Acknowledgements}

The authors acknowledges the support provided by the project n. 23024, INOTEC - Innovative material of ultra-high ductility for the rehabilitation of the built patrimony, co-financed by the European Regional Development Fund (FEDER) through the Operational Program COMPETE. The collaboration provided by Owenscorning (glass fibers), Exporplas (polypropylene fibers), Fisipe (PAN Fibers), Sika (superlasticizers), Pegop (Fly ash) and PIEP (in the preparation of the connectors) are acknowledged.

\section{References}

[1] European Committee for Standardization, Eurocode 8: Design of structures for earthquake esistance Part 1: General rules, seismic actions and rules for buildings, vol. NP EN 1998-1. 2010.

[2] E. Vintzeleou and T. P. Tassios, "Seismic behaviour and design of infilled RC frames," Int. J. Earthq. Eng. Eng. Seismol., vol. 3, no. 2, pp. 22-28, 1989.

[3] P. Thomas and M. J. Priestley, Seismic design of reinforced concrete and masonry buildings. New York Wiley-Interscience, America, 1992.

[4] A. Dehghani, G. Fischer, and F. Nateghi Alahi, "Strengthening masonry infill panels using engineered cementitious composites," Mater. Struct., vol. 48, no. 1-2, pp. 185-204, Jan. 2015.

[5] C. V. R. Murty and S. K. Jain, "Beneficial influence of masonry infill walls on seismic performance of RC frame buildings," in 12th World Conference on Earthquake Engineering (Auckland, New Zealand, January 30), 2000.

[6] T. Valente, D. Gonçalves, C. Frazão, and J. A. Barros, "Argamassa de ultra-elevada ductilidade para reabilitação: comportamento mecânico e durabilidade," in $5^{a} s$ Jornadas Portuguesas de Engenharia de Estruturas, JPEE 2014, Lisboa, Portugal, 2014. 
[7] European Committee for Standardization, Eurocode 4: Design of composite steel and concrete structures Part 1-1: General rules and rules for buildings, vol. EN 1994-1-1. 2010. 